University of Nebraska - Lincoln

DigitalCommons@University of Nebraska - Lincoln

Ralph Skomski Publications

Research Papers in Physics and Astronomy

2008

Ordered Layers of Co Clusters on BN Template Layers

J. Zhang

Max-Planck-Institut für Festkörperforschung

V. Sessi

Max-Planck-Institut für Festkörperforschung

C. H. Mlchaelis

Max-Planck-Institut für Festkörperforschung

I. Brihuega

Max-Planck-Institut für Festkörperforschung

Jan Honolka

Max-Planck-Institut für Festkörperforschung, honolka@fzu.cz

See next page for additional authors

Follow this and additional works at: https://digitalcommons.unl.edu/physicsskomski

Part of the Physics Commons

Zhang, J.; Sessi, V.; Mlchaelis, C. H.; Brihuega, I.; Honolka, Jan; Kern, K.; Skomski, Ralph; Chen, X.; Rojas, Geoffrey; and Enders, Axel, "Ordered Layers of Co Clusters on BN Template Layers" (2008). Ralph Skomski Publications. 56.

https://digitalcommons.unl.edu/physicsskomski/56

This Article is brought to you for free and open access by the Research Papers in Physics and Astronomy at DigitalCommons@University of Nebraska - Lincoln. It has been accepted for inclusion in Ralph Skomski Publications by an authorized administrator of DigitalCommons@University of Nebraska - Lincoln. 


\section{Authors}

J. Zhang, V. Sessi, C. H. Mlchaelis, I. Brihuega, Jan Honolka, K. Kern, Ralph Skomski, X. Chen, Geoffrey Rojas, and Axel Enders 


\title{
Ordered layers of Co clusters on BN template layers
}

\author{
J. Zhang, V. Sessi, C. H. Michaelis, I. Brihuega, J. Honolka, and K. Kern \\ Max-Planck-Institut für Festkörperforschung, Heisenbergstrasse 1, 70569 Stuttgart, Germany \\ R. Skomski, X. Chen, G. Rojas, and A. Enders* \\ Department of Physics and Astronomy and Nebraska Center for Materials and Nanoscience, \\ University of Nebraska, Lincoln, Nebraska 68588, USA
}

(Received 1 August 2008; published 31 October 2008)

\begin{abstract}
The synthesis of highly ordered monolayers of metallic nanoclusters by a buffer-layer assisted growth (BLAG) route is introduced and investigated. The focus is on clusters of Co deposited onto mechanically stable, periodically corrugated boron-nitride layers by repeated BLAG cycles. The approach combines the advantages of well-established preparation methods for surface-supported clusters; namely, the versatility of cluster deposition from the gas phase and the positional accuracy of the directed growth on template surfaces. The particle coverage and geometry are obtained from scanning tunneling microscopy experiments, and analyzed with analytic models and by Monte Carlo simulations. The model shows that the approach to full coverage is critically slowed down by attractive interparticle interaction, which results in the coalescence and growth of some of the clusters. The method represents a generic approach to fabricate ordered layers of clusters of virtually any metal.
\end{abstract}

DOI: $10.1103 /$ PhysRevB.78.165430

PACS number(s): 61.46.Hk, 62.23.Eg, 68.37.Ef, 81.07.Bc

\section{INTRODUCTION}

The creation of nanostructures with well-defined geometry, chemistry, and long-range periodicity is a key challenge in nanomagnetism. This includes but is not limited to information technology, where magnetic thin films have been used to increase the areal storage density of computer hard disks by many orders of magnitude in recent decades. ${ }^{1-3} \mathrm{Fu}-$ ture progress will be based on nanoscale monodisperse and aligned magnetic units, densely packed into an ordered monolayer and with stable remanent magnetization at room temperature, accessible switching fields, and negligible interactions. ${ }^{3}$ This requires the fabrication of particles of typical hard-magnetic materials (magnetic anisotropy $K_{1}$ $\sim 5 \mathrm{MJ} / \mathrm{m}^{3}$ ) with approximately $3 \mathrm{~nm}$ diameter as they exhibit a stable magnetization direction at room temperature. ${ }^{4}$ The magnetization of smaller particles would be thermally unstable, whereas bigger particles would lead to a waste of areal density.

This paper focuses on the positioning of nanoclusters at surfaces as a key challenge in nanotechnology and as a specific requirement in bit-patterned magnetic recording. ${ }^{3}$ Experimental approaches for the fabrication of ordered nanoparticle layers center around the deposition of nanoclusters from the liquid phase, ${ }^{5}$ the soft landing from the gas phase, ${ }^{6}$ and the directed self-assembly on template surfaces. ${ }^{7,8}$ While the first two approaches give only little control over the position of the clusters per se, the latter has the advantage that the lateral arrangement of the particles can be controlled precisely by using template surfaces. Such surfaces provide well-defined energetic sinks with subnanometer accuracy to guide the nucleation processes and diffusion of atoms, and to form nanostructures or clusters at specific sites. A number of templates, including reconstructed surfaces, ${ }^{9}$ strain-relief patterns, ${ }^{10}$ or supramolecular architectures,${ }^{11}$ have been used recently to fabricate nanoparticle arrays. ${ }^{12}$ It is generally found that the directed growth works well for very specific film/substrate systems but does not represent a generic approach for the synthesis of ordered nanoparticle layers.

Here, we present an alternative approach that combines the advantages of template-assisted growth with the versatility of cluster deposition. Metal clusters have been fabricated in this work by noble-gas buffer-layer assisted growth (BLAG) (Refs. 13 and 14) directly on template surfaces. Since BLAG permits, in principle, the deposition of any material on any substrate, ${ }^{15,16}$ our approach has the potential to be applicable to many other metallic or nonmetallic materials.

\section{EXPERIMENTAL}

Mechanically stable boron-nitride (BN) monolayers are used as templates for the fabrication of ordered layers of metal cluster. In the past, the use of such BN layers has been limited to the positioning of nonmagnetic molecules, such as $\mathrm{C}_{60}$ (Ref. 17) and naphtalocyanine. ${ }^{18}$ The boron-nitride layer is formed by thermal decomposition of borazine gas, $(\mathrm{HBNH})_{3}$, on $\mathrm{Rh}(111)$ surfaces in an ultrahigh vacuum chamber. ${ }^{17}$ The resulting $h$-BN layers are atomically thin, electrically insulating, chemically inert, mechanically extremely stable, and show a strain-driven hexagonally ordered corrugation with a periodicity of $3.2 \mathrm{~nm},{ }^{18,19}$ as can be seen in the scanning tunneling microscopy (STM) images in Fig. 1. Recent STM and theoretical analysis has shown that the BN layer is buckled due to epitaxial strain and only locally attached to the Rh surfaces. ${ }^{18,20}$ Such BN layers are commonly referred to as nanomesh but they rather resemble the shape of a muffin tin. The difference in height between the attached areas (depressions) and the detached ridges is approximately $0.55 \AA .{ }^{20}$

The cobalt nanoclusters were fabricated directly at the $\mathrm{BN}$ layer by BLAG. The preparation steps include the deposition of $\mathrm{Co}$ on a xenon layer that has been preadsorbed on the BN 


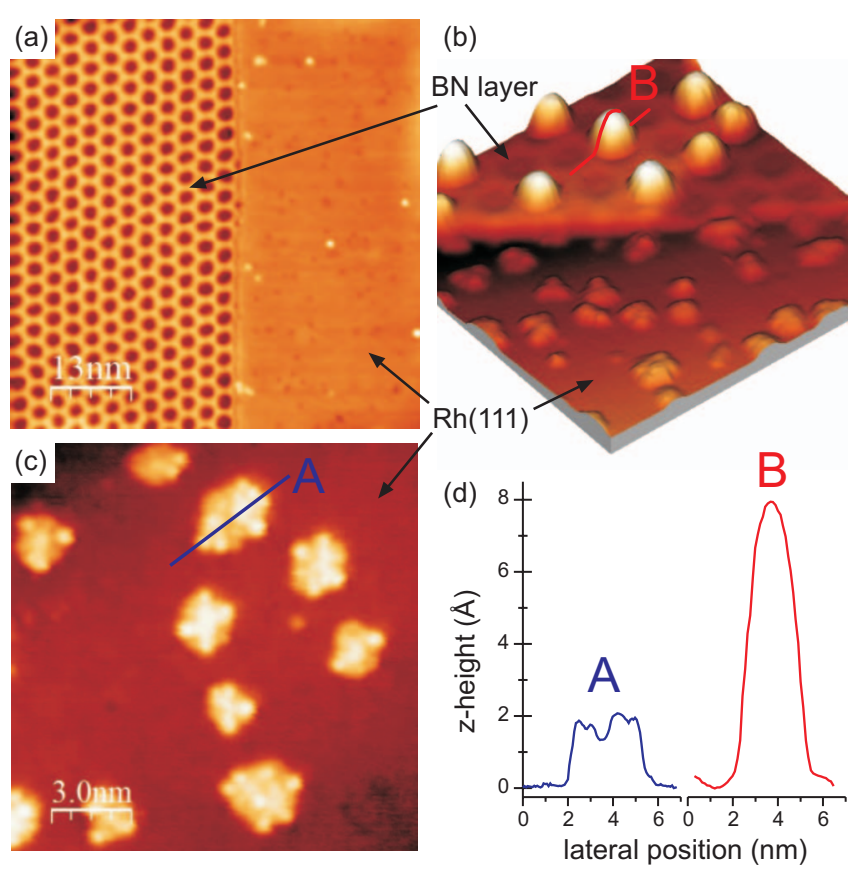

FIG. 1. (Color) (a) STM images of a partial BN nanomesh layer on $\mathrm{Rh}(111)$; (b) Co clusters on a partial nanomesh layer as in (a), deposited with one BLAG cycle; (c) Co clusters on $\mathrm{Rh}(111)$. (d) Line profiles of Co clusters in contact with $\mathrm{Rh}(111)(\mathrm{A})$ and $\mathrm{BN}$ (B).

layer at $35-40 \mathrm{~K}$, followed by Xe desorption, to bring the clusters in contact with the substrate. Clusters of Co form directly on the Xe due to the high mobility of the metal atoms on Xe even at such low temperatures. The metal clusters on top of the Xe become highly mobile and coalesce during Xe desorption, thus growing in size until making contact with the surface. The final cluster size depends on the Xe thickness and on the metal coverage. ${ }^{21,22}$ Clusters of 1-3 nm in diameter are achieved by using approximately 2 monolayers (ML) of Xe and 3\%-5\% of a full monolayer of Co.

Figures 1(a) and 1(b) show STM images of an incomplete boron-nitride layer, before and after cobalt-cluster deposi- tion. Incomplete $\mathrm{BN}$ layers are fabricated by exposure of the hot $\mathrm{Rh}(111)$ surface to less than 20 langmuirs (L) [1 langmuir $=10^{-6}$ Torr sec $]$ of borazine gas, and have been chosen for this study to demonstrate the template effect of the $\mathrm{BN}$. The clusters on the pristine Rh surface are randomly distributed but on the BN layer they preferentially occupy the depression sites. Furthermore, line scans through the clusters on the Rh and BN layers, such as those shown in Fig. 1(d), reveal striking particle-shape differences on the $\mathrm{Rh}$ and $\mathrm{BN}$ layers. On the $\mathrm{BN}$ layer, the Co clusters appear to have a smooth, hemispherical shape but on the $\mathrm{Rh}(111)$, they form corrugated islands of 1.5-2 $\AA$ average apparent height. We ascribe this difference to the wetting behaviors of the layers. The clusters remain hemispherical on the BN layer as a result of dewetting, whereas surface wetting results in Co islands of monolayer and bilayer heights on pristine $\mathrm{Rh}$ surface areas. The use of a noble-gas layer during preparation is crucial in achieving a template effect for metals with the BN layer. Direct deposition of Co or other metals on the BN layer at 80 or $300 \mathrm{~K}$ resulted in disordered cluster layers.

With the deposition parameters $\theta_{\mathrm{Co}}=0.05 \mathrm{ML}$ and $\Theta_{\mathrm{Xe}}$ $=5 \mathrm{~L}$, about $30 \%$ of the depressions in the $\mathrm{BN}$ layer are filled by one BLAG cycle, as shown in the STM image in Fig. 2(a). The number of the clusters deposited per area can be controlled, to some extent, by varying experimental parameters but depression occupancies beyond $40 \%$ are difficult to achieve in a single BLAG cycle. In order to fabricate ordered cluster layers with BN layer occupations approaching the ideal value of $100 \%$ (full coverage), the BLAG cycles were repeated several times. The fraction of occupied depressions gradually increases, and after three subsequent BLAG cycles approximately $70 \%$ of the depressions are occupied [Fig. 2(c)]. Figure 2(d) shows the occupation of the nanomesh as function of the number $m$ of deposition cycles for two samples: $3 \%$ of a ML Co on $5 \mathrm{~L} \mathrm{Xe} \mathrm{(red)} \mathrm{and} 5 \%$ of a ML Co on $5 \mathrm{~L} \mathrm{Xe} \mathrm{(blue)} \mathrm{per} \mathrm{BLAG} \mathrm{cycle.} \mathrm{It} \mathrm{appears} \mathrm{that}$ larger amounts of Co per cycle help in filling the BN layer more quickly. However, more Co per cycle results also in larger clusters, and we observe that the presence of larger clusters impedes their ordered arrangement. This might be
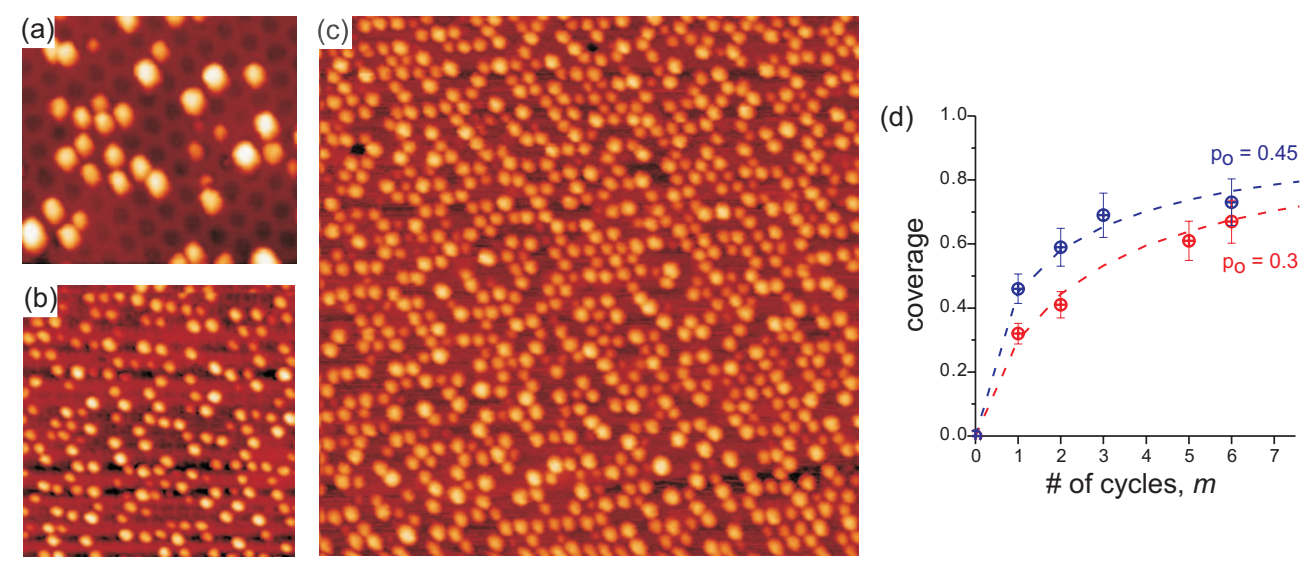

FIG. 2. (Color) Increase in the occupation of the BN layer with Co clusters by repeated BLAG cycles. (a) STM images after one BLAG cycle $(5 \% \mathrm{Co} / 5 \mathrm{~L} \mathrm{Xe})\left(30 \times 30 \mathrm{~nm}^{2}\right)$, (b) after two BLAG cycles $\left(50 \times 50 \mathrm{~nm}^{2}\right)$, and (c) after three BLAG cycles $\left(100 \times 100 \mathrm{~nm}^{2}\right)$, and $(\mathrm{d})$ analysis of the $\mathrm{BN}$ occupation for $3 \%$ of a ML Co/5 L Xe per cycle (red) and $5 \%$ of a ML Co/5 L Xe per cycle (blue). The dashed lines are from an analytical model in Eq. (2) with $\lambda=1.05$ and values of $p_{0}$ corresponding to the coverages at $m=1$. 

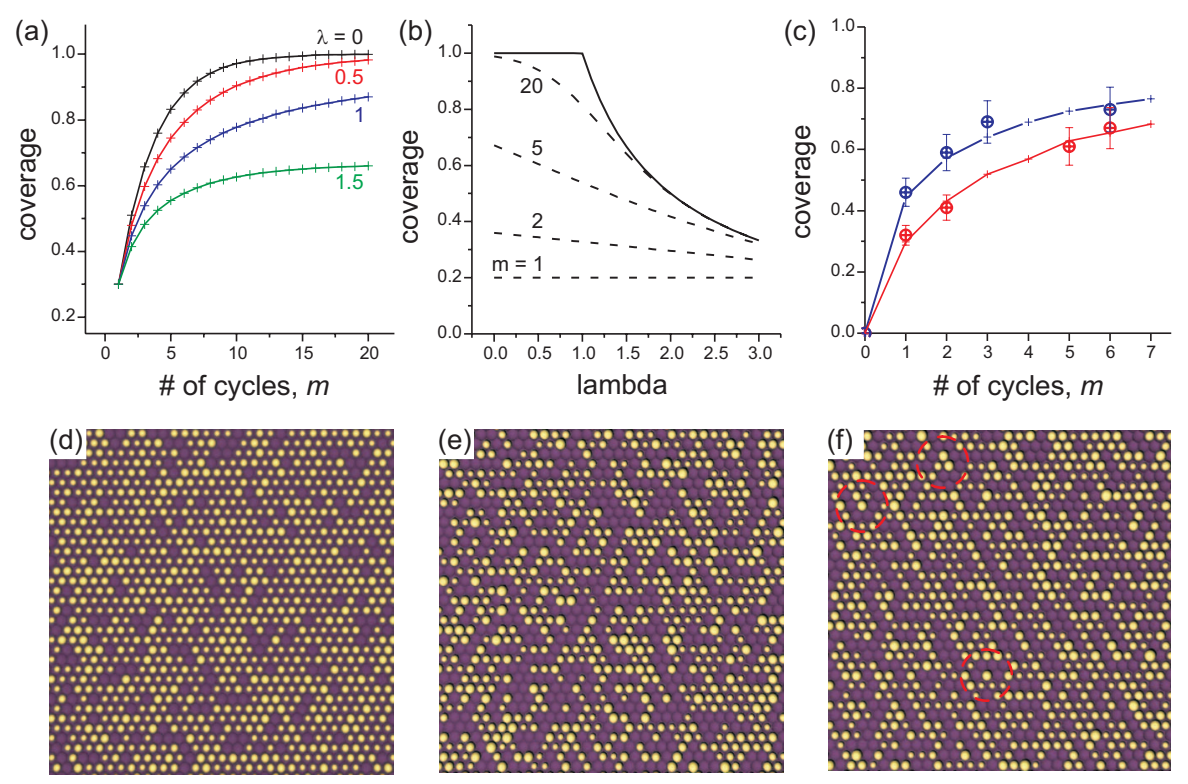

FIG. 3. (Color) Models of the BN occupation. [(a) and (b)] Analytical model, assuming attractive interaction between clusters, which leads to a slowing down of the BN layer filling for $\lambda>0$ and a decrease in the maximum possible cluster coverage for $\lambda>1$. [(c)-(f)] Monte Carlo simulations of the BN occupation. (c) The experimental data from Fig. 2(c) can be reproduced by assuming exponential volume dependence of the attraction parameter $\lambda, V_{C}=5.0, c_{0}=0.3$ (red), and $c_{0}=0.45$ (blue). Visualization of the BN layer occupation with clusters (yellow), (d) without cluster hopping to a neighboring site, (e) with constant hopping probability, and (f) a hopping probability that depends exponentially on the volume of the neighboring cluster (see text). Encircled in red are some clusters that grew large by merging with their neighbors.

due to decreased mobility of the clusters with increased volume, or simply a geometrical on-site repulsion problem if the clusters' diameters exceed the distance between the centers of neighboring depressions on the $\mathrm{BN}$ layer.

\section{MODELING AND SIMULATION}

To analyze the cluster coverage as a function of the number of deposition cycles and the real-space distribution of the particles, we have used analytical model calculations and Monte Carlo (MC) simulations. In the simplest model, the particles arriving on the surface occupy a fraction $p$ of the empty depressions so that the coverage $c_{m}$ after the $m$ th cycle is given by the discrete rate equation,

$$
c_{m+1}=c_{m}+p\left(1-c_{m}\right) .
$$

Subject to the initial condition $c_{o}=0$, the solution of this equation is $c_{m}=1-(1-p)^{m}$. By considering $m=\infty$, we see that this equation predicts an approach to complete coverage $\left(c_{\infty}=1\right)$, which seems in contradiction to the experiment [Fig. 2(c)]. Physically, the arriving particles tend to be attracted by occupied pits (clusters). Little is known about the details of this attraction but it probably reflects the mobility of the particles in the noble-gas matrix and at the surface during Xe desorption. We will see that the effect exhibits a subtle dependence on an attraction parameter $\lambda$ so that magnetic interactions between particles may be important, as well.

To describe the attraction effect, we assume that the probability $p$ depends on the coverage of the nanomesh. A certain fraction of the particles that arrive at empty depressions are diverted toward filled ones. This means that some of the deposited clusters coalesce with newly deposited clusters during a BLAG cycle and thus grow in size. In the present model, we assume that this effect is linear in the coverage, $p=p_{0}\left(1-\lambda c_{m}\right)$. Equation (1) now becomes

$$
c_{m+1}=c_{m}+p_{0}\left(1-\lambda c_{m}\right)\left(1-c_{m}\right) \text {. }
$$

In real space, $\lambda$ describes an excluded volume around occupied sites (clusters) and a large $\lambda$ means that deposited clusters are very efficient in capturing particles arriving in their vicinity and preventing the occupancy of neighboring empty sites. We observe experimentally that the number of deposited clusters always increases monotonically, which means that $\lambda$ is always $<1 / p_{0}$.

Equation (2) is solved very easily by numerical iteration. Figure 3(a) shows the calculated coverage as a function of the number $m$ of deposition cycles. For small concentrations $c_{m}$, that is, for small $p_{0}$ or $m$, the solution of Eq. (2) is exponential. However, the extrapolated maximum coverage is reduced, $c_{\infty}=1 /(1+\lambda)$, and the approach to saturation is slower. Qualitative deviations from Eq. (1) occur as $c_{\mathrm{m}}$ approaches $c_{\infty}$, and the extrapolated value $c_{\infty}=1 /(1+\lambda)$ is actually a very poor estimate. Figure $3(\mathrm{~b})$ shows the coverage as a function of the attraction parameter. According to Eq. (2) the $c_{m}$ cannot increase further if it becomes $c_{m}=1 / \lambda$. The $\lambda$ defines thus also a critical coverage above which all of the clusters arriving at the surface during a BLAG cycle are assimilated by previously deposited clusters. We see therefore critical behavior at $\lambda=1$. For values $\lambda<1$, the occupied 
pores reduce the deposition rate but leave the maximum coverage unchanged $\left(c_{\infty}=1\right)$. For $\lambda>1$, the maximum coverage becomes $<1$. This is of great practical importance because it means that seemingly small changes in deposition conditions can make the difference between complete and incomplete coverages. Matching the experimental data of the BN coverage in Fig. 2(d) with the function in Eq. (2) requires $\lambda$ $=1.05$; thus the maximum possible coverage for this system under the present preparation conditions is $c_{\infty}=0.95$.

The case $\lambda=1$ exhibits an interesting slowing down as the coverage approaches $c_{\infty}=1$. The slow approach to saturation is clearly visible in Fig. 3(a), where both the black and the red lines approach $c_{\infty}=1$ but the red line exhibits a nonexponential convergence. By putting $\lambda=1$ in Eq. (2), it is easy to show that the approach to saturation obeys the power law $p_{0}\left(1-c_{m}\right)=1 / m$. This equation is obtained by putting $\lambda=1$ in Eq. (2), replacing $c_{m+1}-c_{m}$ by $d c / d m$, and integration of the resulting differential equation. For $\lambda$ values slightly smaller than one, the system exhibits a preasymptotic powerlaw approach, followed by an exponential approach to saturation with an effective deposition rate $p_{\text {eff }}=(1-\lambda) p_{0}$. In other words, the coverage increases slowly but approaches full coverage in an exponential manner. Note that the present regimes are different from well-known mechanisms such as Langmuir and Brunauer-Emmett-Teller (BET) adsorptions. ${ }^{23}$ The latter are based on equilibrium thermodynamics, whereas the present absorption mechanism is partially of the first-passage type-where particles are allowed to stick to each other so that only a part of the full phase space is explored.

The model of Eq. (2) describes the coverage as a function of the number $m$ of deposition cycles but does not explain the nanomesh occupation qualitatively. To reproduce the effect of the attractive cluster-cluster interaction on the lateral distribution, we have used a Monte Carlo simulation. It is assumed that an integer number $p_{0}$ of clusters of unit volume are randomly distributed onto the discrete positions of a hexagonal lattice with each deposition cycle. After assigning those clusters to preliminary lattice sites, they are treated according to the following two rules. First, if a site is already occupied due to a preceding deposition cycle, then the new cluster is simply attached to the existing cluster, whose size is consequently enlarged by one unit volume. Second, if the site is empty, then the cluster is allowed to hop to a nearestneighbor site $i$ with a certain probability $\eta_{i}$ and to merge with the cluster occupying the neighboring site unless it is empty. This process implies some mobility of the clusters at some point during the deposition and attractive clustercluster interaction, as also assumed in Eq. (2). A direct consequence is that the average cluster size increases linearly with every deposition step, something that can clearly be deduced from the STM images.

Figures 3(d)-3(f) show the results of MC simulations, all after three deposition steps with $p_{0}=0.45$. In Fig. 3(d), no hopping of clusters after deposition was permitted, and the BN layer occupation is $90 \%$. Hopping was permitted in the simulation in Fig. 3(e), based on the simple constantprobability rule that a cluster merges with a randomly chosen cluster in its nearest neighborhood. Figure 3(e) shows the result of a simulation using $p_{0}=0.45$ and the probability $\eta$ $=0.65$, as these parameters give best agreement with the experimental data for $c(m)$ in Fig. 2(d). Figure 3(f) shows the result of a simulation where $\eta$ was assumed to be dependent on the volume $V_{i}$ of the clusters in its six nearest-neighbor sites $(i=1-6)$. We find that linear and exponential volume dependencies of $\eta$,

$$
\begin{gathered}
\eta_{i}^{\text {linear }}=\left(V_{i}+n\right) / \sum_{k=1}^{6}\left(V_{k}+n\right), \\
\eta_{i}^{\exp }=\exp \left(\frac{V_{i}}{V_{C}}\right) / \sum_{k=1}^{6} \exp \left(\frac{V_{k}}{V_{C}}\right),
\end{gathered}
$$

give similar results. In Eq. (3), $V_{i}$ is the volume of the $i$ th cluster in direct neighborhood of a newly deposited cluster, $V_{c}$ is a critical volume, and $n$ is a constant. Both $V_{c}$ or $n$ can be used as fit parameters to describe the experimental data [Fig. 3(c)]. The BN coverages in Figs. 3(e) and 3(f) are 0.63 and 0.64 , respectively. The radii $r$ of the circles representing the clusters in Fig. 3 reflect their volume according to $r$ $\sim V^{1 / 3}$. While the integral cluster volume is the same in all three simulations [Figs. 3(d)-3(f)], the BN layer occupation is visibly reduced by the introduction of attractive cluster interaction.

The simulation based on the volume-dependent assimilation model reproduces the experimentally found coverage $c_{\mathrm{m}}$ [Fig. 3(c)], the formation of empty channels of one depression diameter in width on the $\mathrm{BN}$, the existence of very large, isolated clusters, and the measured cluster size as function of $m$ (not shown). As far as the $c_{m}$ is concerned, the MC predictions are very similar to those derived from Eq. (2) [compare Figs. 2(d) and 3(c)].

\section{CONCLUDING REMARKS}

The present structure has several specific advantages. First, the clusters' center-to-center distance of order of $3 \mathrm{~nm}$ is ideal for application as patterned media for ultrahigh density magnetic data storage (one cluster per bit). Second, the structures have a high periodicity, and some aperiodicity can actually be accounted for by multiple-pass reading and writing. Third, the mesh is suitable for robust annealing at high temperatures, which is an important practical consideration for FePt based recording media. ${ }^{4}$ Besides being a template, the BN layer has another trait: it decouples deposited nanostructures electronically from the metal substrate. Tunneling spectroscopy studies revealed a Coulomb gap of $>160 \mathrm{meV}$ in the electronic structure of the Co clusters on the BN layer, proving their electronic decoupling from the rhodium substrate. ${ }^{19} \mathrm{BN}$ layers thus turn out to be an ideal playground for the study of the electronic interaction of nanostructures with the substrate.

The presented buffer-layer assisted growth on boronnitride template surfaces combines the versatility of the cluster deposition from the gas phase with the positional accuracy of directed, self-assembled growth. It represents a promising and viable strategy for the fabrication of ordered 
nanodot layers of virtually any material. We have demonstrated that as much as $70 \%$ of the $\mathrm{BN}$ layer template is occupied with Co clusters after only 3 cycles of buffer-layer assisted growth on boron-nitride templates. Higher BN layer filling seems possible by continued BLAG cycles, and the key to success for achieving complete mesh occupation will be in reducing mutual interactions between clusters and the cluster mobility by the preparation conditions.

\section{ACKNOWLEDGMENTS}

We thank Armando Rastelli for providing the IDL code for the statistical analysis, and Markus Weinmann for the synthesis of the borazine gas. We acknowledge financial support from the Humboldt Foundation, DFG (SSP 1153), NSF CAREER (Contract No. DMR-0747704), and NSF MRSEC (Contract No. DMR-0213808).
*Corresponding author; a.enders@me.com

${ }^{1}$ B. Hayes, Am. Sci. 90, 212 (2002).

${ }^{2}$ S. D. Bader, Rev. Mod. Phys. 78, 1 (2006).

${ }^{3}$ D. Weller and T. McDaniel, in Advanced Magnetic Nanostructures, edited by D. Sellmyer and R. Skomski (Springer, Berlin, 2006), pp. 295-324.

${ }^{4}$ Ch. Rong, N. Poudyal, G. S. Chaubey, V. Nandwana, R. Skomski, Y. Q. Wu, M. J. Kramer, and J. P. Liu, J. Appl. Phys. 102, 043913 (2007).

${ }^{5}$ S. Sun, C. B. Murray, D. Weller, L. Folks, and A. Moser, Science 287, 1989 (2000).

${ }^{6}$ K. Bromann, C. Felix, H. Brune, W. Harbich, R. Monot, J. Buttet, and K. Kern, Science 274, 956 (1996).

${ }^{7}$ J. V. Barth, G. Constantini, and K. Kern, Nature (London) 437, 671 (2005).

${ }^{8}$ A. Enders, P. Gambardella, and K. Kern, in The Handbook of Magnetism and Advanced Magnetic Materials, Fundamentals and Theory Vol. 1, edited by H. Kronmüller and S. Parkin (Wiley, Chichester, UK, 2006), pp. 577-598.

${ }^{9}$ O. Fruchart, P.-O. Jubert, C. Meyer, M. Klaua, J. Barthel, and J. Kirschner, J. Magn. Magn. Mater. 239, 224 (2002).

${ }^{10}$ H. Brune, M. Giovannini, K. Bromann, and K. Kern, Nature (London) 394, 451 (1998).

${ }^{11}$ J. A. Theobald, N. S. Oxtoby, M. A. Phillips, N. R. Champness, and P. H. Beton, Nature (London) 424, 1029 (2003).
${ }^{12}$ W. Chen and A. T. S. Wee, J. Phys. D 40, 6287 (2007).

${ }^{13}$ J. H. Weaver and G. D. Waddill, Science 251, 1444 (1991).

${ }^{14}$ L. Huang, S. J. Chey, and J. H. Weaver, Phys. Rev. Lett. 80, 4095 (1998).

${ }^{15}$ J. H. Weaver and V. N. Antonov, Surf. Sci. 557, 1 (2004).

${ }^{16}$ G. Kerner and M. Asscher, Surf. Sci. 557, 5 (2004).

${ }^{17}$ M. Corso, W. Auwaerter, M. Muntwiler, A. Tamai, T. Greber, and J. Osterwalder, Science 303, 217 (2004).

${ }^{18}$ S. Berner, M. Corso, R. Widmer, O. Groening, R. Laskowski, P. Blaha, K. Schwarz, A. Goriachko, H. Over, S. Gsell, M. Schreck, H. Sachdev, T. Greber, and J. Osterwalder, Angew. Chem., Int. Ed. 46, 5115 (2007).

${ }^{19}$ I. Brihuega, C. H. Michaelis, J. Zhang, S. Bose, V. Sessi, J. Honolka, M. A. Schneider, A. Enders, and K. Kern, Surf. Sci. 602, L95 (2008).

${ }^{20}$ R. Laskowski, P. Blaha, T. Gallauner, and K. H. Schwarz, Phys. Rev. Lett. 98, 106802 (2007).

${ }^{21}$ D. Repetto, J. Honolka, S. Rusponi, H. Brune, A. Enders, and K. Kern, Appl. Phys. A: Mater. Sci. Process. 82, 109 (2006).

${ }^{22}$ J. Zhang, D. Repetto, V. Sessi, J. Honolka, A. Enders, and K. Kern, Eur. Phys. J. D 45, 515 (2007).

${ }^{23}$ S. Brunauer and L. E. Copeland, in Handbook of Physics, edited by E. U. Condon and H. Odishaw (McGraw-Hill, New York, 1958), Pt. 5, Chap. 7. 